\title{
Complejidad y tipos de conflictividad en la tenencia de la propiedad en la Costa Caribe de Nicaragua
}

\section{Complexity and types of conflict in property ownership on the Caribbean Coast of Nicaragua}

\author{
Sandra Carolina Rojas Hooker ${ }^{1}$
}

\section{Resumen}

Este artículo se basó en el estudio "Tipos de tenencia de la propiedad en la Costa Caribe de Nicaragua" con énfasis en la complejidad y tipos de conflictividad en la tenencia de la propiedad. Para ello, se analizan los diferentes tipos de propiedad teniendo como base los principios y categorías contenidas en la Carta Magna, las leyes generales y especiales relacionadas con la temática.

La metodología fue cualitativa encaminada al reconocimiento de los niveles de complejidad y tipos de conflictividad que se presentan según las formas de propiedad reconocidas en la legislación nacional. Para ello, se realizó grupos focales y entrevistas con la participación de representantes de las Alcaldías Municipales, representantes de los gobiernos indígenas y afrodescendientes, y demás sectores sociales, así como la revisión documental.

Las principales complejidades son La creciente población no indígena en territorios indígenas, la politización y partidización en la elección de las autoridades comunitarias, el desfase y la falta de conclusión de la etapa de saneamiento que impide la emisión de los títulos de propiedad a favor de las comunidades indígenas y consecuentemente dificulta las negociaciones para la inversión.

Palabras clave: Tenencia de la propiedad; formas de propiedad; derechos colectivos; niveles de complejidad; tipos de conflictividad.

\section{Abstract}

This article was based on the study "Types of property ownership in the Caribbean Coast of Nicaragua" with an emphasis on the complexity and types of conflict in property ownership. For this, the different types of property are analyzed based on the principles and categories contained in the Magna Carta, the general and special laws related to this subject.The methodology was qualitative aimed at recognizing the levels of complexity and types of conflict that occur according to the forms of ownership recognized in national legislation. For this, focus groups and interviews were carried out with the participation of representatives of the Municipal Governments, representatives of indigenous and Afro-descendant governments, and other social sectors, as well as a documentary review in order to have a comprehensive look at this issue.

The main complexities are the growing non-indigenous population in indigenous territories, the politicization and partisanship in the election of community authorities, the lag and the lack of completion of the sanitation phase that prevents the issuance of property titles in favor of the indigenous communities and consequently makes investment negotiations difficult.

Keywords: Property Ownership; forms of ownership; collective rights; complexity levels; types of conflict.

\footnotetext{
1 Máster en Estudios Indígenas. Directora Instituto de Estudios y Promoción de la Autonomía de la URACCAN. Correo: sandra.rojas@uraccan.edu, ni; ORCID https://orcid.org/0000-0002-7467-2366
}

Recibido: 06/10/2020 - Aprobado: 14/11/2020 


\section{Introducción}

La Costa Caribe de Nicaragua, con su diversidad étnica y cultural ocupa aproximadamente el 50\% del territorio nacional con una extensión territorial de $60,329.90 \mathrm{~km}^{2}$. Desde 2007 a noviembre 2016 se han titulado 23 territorios, 16 en la Costa Caribe Norte, 3 en la zona de régimen especial (Jinotega) y 4 en la Costa Caribe Sur que corresponden a 304 comunidades. El total del área demarcada y titulada es de $37,841.99 \mathrm{~km} 2$, equivalente a 31.6\% del territorio nacional. En la Costa Caribe de Nicaragua habita el 15\% de la población total del país, lo que la convierte en la segunda región más poblada de Nicaragua, después del departamento de Managua. En ella reside la mayor parte de los pueblos indígenas y afrodescendientes que enriquecen y aportan de manera significativa al desarrollo con identidad.

Este artículo aborda los niveles de complejidad y tipos de conflictividad en la tenencia de la propiedad en la Costa Caribe Nicaragüense. El interés de este trabajo viene dado por el incremento de terceros en territorios indígenas y afrodescendientes en los últimos años presentado una serie de conflictos al no saber exactamente las condiciones reales en las que se está adquiriendo una propiedad. Por tanto, este trabajo es uno de los primeros que persigue analizar el estado actual en que se encuentra la tenencia de la propiedad y las posibles soluciones planteadas para brindar seguridad jurídica a las partes.

Las técnicas de investigación fueron la revisión documental, las entrevistas semi-estructuradas y los grupos focales. La revisión documental se efectuó principalmente en Bilwi, Siuna, Bluefields y Managua. Los grupos focales fueron ejecutados en las cabeceras municipales de Puerto Cabezas, Siuna, Bluefields y Nueva Guinea, con la participación de 45 personas.

Los pueblos indígenas y afrodescendientes tienen formas de vida únicas, y su cosmovisión se basa en su estrecha relación con la tierra. Las tierras tradicionalmente utilizadas y ocupadas por ellos son un factor primordial de su vitalidad física, cultural y espiritual, arraigada en su historia. Esta relación única con el territorio tradicional puede expresarse de distintas maneras, dependiendo de sus particularidades y circunstancias específicas. Existe una conflictividad respecto a la tenencia de la tierra sea propiedad privada o pública porque existen un alto nivel de inseguridad jurídica de la propiedad que es el factor determinante en los niveles de complejidad de la propiedad privada en la Costa Caribe. La consecuencia es que la mayoría de los ocupantes de propiedades rurales no tienen derechos de propiedad o sus derechos de posesión son precarios. El punto más alto en este tensionamiento gira alrededor de la tenencia de la tierra y sus recursos y en muchas ocasiones ha desembocado en quemas de viviendas, destrucción de siembras, lesiones y muertes.

Respecto a la autonomía de la Costa Caribe y los derechos de los pueblos originarios, el artículo 5 de la Constitución Política de Nicaragua entre otros principios se reconoce el derecho de los pueblos indígenas y afrodescendientes de su propia identidad dentro de un Estado unitario e indivisible, el reconocimiento a las distintas formas de propiedad tales como: pública, privada, asociativa, cooperativa, comunitaria, comunal, familiar y mixta. Por tanto, el territorio constituye un referente central para comprender las dinámicas jurídicas, políticas y sociales que han marcado la relación del Estado con los pueblos indígenas y afrodescendientes y de estas entre sí.

En síntesis, es necesario coordinar con la Corte Suprema de Justicia el establecimiento de mecanismos para la agilización de los trámites registrales de acuerdo con la ley vigente y, sobre todo, el traslado de asientos registrales pendientes del Registro Público de la Propiedad Inmueble y Mercantil de Bluefields hacia el Registro de la Propiedad de Puerto Cabezas, para evitar la doble inscripción y dar mayor seguridad jurídica. Asimismo, realizar un proceso de depuración del Registro de la Propiedad para cancelar todos los títulos de terrenos inscritos a favor de particulares en territorios indígenas, afrodescendientes y áreas públicas para tener un inventario y registro actualizado de la propiedad.

Se recomienda realizar las coordinaciones con los distintos niveles de gobiernos para iniciar las negociaciones de cara a la implementación de la etapa de saneamiento de territorios indígenas y afrodescendientes, 
sobre todo, en aquellos lugares donde se presentan mayores niveles de complejidad y conflictividad como los señalados en el presente artículo.

\section{Revisión de la literatura}

El derecho a la propiedad tiene una importancia singular para los pueblos indígenas y afrodescendientes porque la garantía del derecho a la propiedad territorial es la base fundamental para el desarrollo de su cultura, su vida espiritual, su integridad y supervivencia económica arraigada en su historia.

El territorio es considerado el espacio vital para los pueblos indígenas y tribales para desarrollar sus actividades culturales y, ligadas a esta, la economía, la política, lo social y lo sagrado; por ende, no es el bien inmaterial individual sino el bien de propiedad colectiva que, lleno de significados, reivindique sus tradiciones y el legado de sus ancestros, para dar un auténtico valor a su cultura.

El derecho a la propiedad en el artículo 21 de la Convención Americana sobre Derechos Humanos tiene importancia para los pueblos indígenas y tribales, porque la garantía del derecho a la propiedad territorial es una base fundamental para el desarrollo de la cultura, la vida espiritual, la integridad y la supervivencia económica de las comunidades indígenas. En Nicaragua los pueblos indígenas y afrodescendientes tienen esta garantía con el artículo 5 de la Constitución Política que, reconoce las distintas formas de propiedad: pública, privada, asociativa, cooperativa, comunitaria, comunal, familiar y mixta, constituyendo el territorio un referente central para comprender las dinámicas jurídicas, políticas y sociales que marcan la relación del Estado con los pueblos y de estas entre sí.

\section{Materiales y métodos}

El estudio es de tipo descriptivo, cualitativo y con el soporte de la información cuantitativa disponible. Las principales técnicas de investigación utilizadas fueron la revisión documental, las entrevistas semi-estructuradas y los grupos focales.

La revisión documental se efectuó principalmente en Bilwi, Siuna, Bluefields y Managua. Los grupos focales fueron ejecutados en las respectivas cabeceras municipales de Puerto Cabezas, Siuna, Bluefields y Nueva Guinea, con la participación de 45 personas. En total se realizaron cuatro entrevistas grupales a 23 miembros de las diferentes Juntas Directivas de los Gobiernos Territoriales y 20 entrevistas directas a funcionarios públicos, notarios y otras personalidades.

\section{Resultados y discusión}

\section{Principales niveles de complejidad y tipos de conflictividad}

La propiedad desde el punto de vista jurídico es: "el dominio que un individuo tiene sobre una cosa determinada, con la que puede hacer lo que desee su voluntad"2. El poder directo e inmediato sobre un objeto o bien, por la que se atribuye a su titular la capacidad de disponer del mismo, sin más limitaciones que las que imponga la ley. Es el derecho real que implica el ejercicio de las facultades jurídicas más amplias que el ordenamiento jurídico concede sobre un bien.

La Declaración Universal de Derechos Humanos reconoce el derecho a la propiedad individual y colectiva y a no ser despojado de ella arbitrariamente. El Código Civil de Nicaragua en su artículo 615 señala que la propiedad es el derecho de gozar y disponer de una cosa, sin más limitaciones que las establecidas por las leyes.

A continuación, detallamos la complejidad y tipos de conflictividad que se presentan en la tenencia de la propiedad en la Costa Caribe.

\footnotetext{
2 Cabanellas de Torres, 1992.
} 


\section{Niveles de complejidad}

\section{Propiedad privada}

Un alto nivel de inseguridad jurídica de la propiedad es el factor determinante en los niveles de complejidad de la propiedad privada en la Costa Caribe. La mayoría de los ocupantes de propiedades rurales no tienen derechos de propiedad o sus derechos de posesión son precarios.

Según la legislación nicaragüense, los títulos de dominio pleno emanados del Estado son de tres tipos: Títulos de reforma agraria, títulos agrarios y títulos reales. Hasta años recientes, la mayoría de los supuestos propietarios privados no tenían documentos legales de propiedad y su ocupación estaba respaldada por documentos extendidos en papel común conocidos popularmente como "papelitos"; otro tanto, respaldado por el acuerdo verbal. Estos "papelitos" tienen un valor entre partes y han prevalecido por muchos años. En caso de conflicto, sirven ante el Juez como un principio de prueba por escrito y cuyo valor para probar posesión debe tomarse como complemento de la prueba testifical.

Con el incremento gradual de la oferta de servicios de notariado, ahora es común la protocolización de documentos privados que no tienen tampoco el valor de documento público. Los notarios también emiten escrituras públicas de cesión de derechos posesorios, declaración de posesión, por mencionar algunos.

La gran cantidad de "papelitos" existentes es un indicativo de que la gente asigna una desproporcionada importancia a cualquier documento para creer que lo que tiene es suyo. Sin embargo, han fallado en fortalecer su seguridad jurídica al no realizar los trámites establecidos para obtener títulos supletorios; a partir de la adquisición inicial de derechos de propiedad por medio de la ocupación y, posteriormente optar a la prescripción adquisitiva o usucapión, después de una posesión prolongada y exenta de vicios.

Se presenta a la vista un primer conflicto: ¿cómo saber exactamente si estás comprando o no un bien? Como este tipo de documentos no es inscribible, se recomienda hacer una investigación in situ para conocer las opiniones de los vecinos y de las autoridades locales y poder un comprar bien, un derecho sobre un terreno no litigioso cuya posesión se puede perfeccionar en el tiempo.

\section{Propiedad pública}

La propiedad pública en la Costa Caribe está constituida principalmente por:

i) Una parte de la extensión superficial de las áreas protegidas.

ii) Las tierras nacionales no ocupadas o en posesión precaria.

iii) Las tierras ejidales o las que se encuentran bajo la administración de las Alcaldías Municipales.

De conformidad con la legislación del caso, en Nicaragua existen nueve categorías de áreas protegidas con algunas directrices para su administración en la que destacan diversas prohibiciones y limitaciones derivadas de los derechos del Estado con respecto a la propiedad privada.

Las variantes de propiedad o posesión de tierras en las áreas protegidas se pueden clasificar en los siguientes grupos:

i. Tierras ocupadas por indígenas en forma ancestral y que, en la actualidad, en su casi totalidad están respaldadas con títulos de territorios indígenas.

ii. Tierras de propiedad privada adquirida antes de la publicación del Reglamento de Áreas Protegidas. La forma de acceder a los derechos de legalización varía según las directrices administrativas de las categorías de áreas protegidas. 
iii. Tierras de dominio público, acreditas con los respectivos títulos.

iv. Ocupantes particulares con derechos precarios o sin derechos de propiedad.

\section{a. Áreas protegidas}

Según el Sistema Nacional de Áreas Protegidas (SINAP) en la Costa Caribe hay 21 áreas protegidas: 17 Reservas Naturales, 2 Reservas Biológicas, 1 Reserva de Biosfera y 1 Parque Nacional con sus directrices administrativas que en su mayoría no permiten actividades agropecuarias y prohíben actividades de exploración y explotación minera, petrolera, concesiones forestales y pesqueras.

Los particulares que estuvieren asentadas en algunas de las nueve categorías de áreas protegidas, antes de marzo de 1999, podrían legalizar su situación, reconociéndoles algunos derechos subordinados a las respectivas directrices administrativas, manteniendo la titularidad el Estado. La situación opuesta es más generalizada, y con mayores niveles de complejidad, pues quienes llegaron después de marzo de 1999, son precaristas, no tienen derechos posesorios y podrían ser sujetos de desalojo, si así lo determina el MARENA.

Es previsible que, al momento de un saneamiento o limpieza registral muchos títulos de propiedad pueden ser anulados y quedará evidenciada su ausencia de legalidad. Esto creará un clima de gran descontento social e inclusive violencia, dado el mayor o menor grado de inversiones realizados por los afectados ya que, en la práctica, ha existido un acceso continuo de personas a las áreas protegidas, sin que el Estado lo impida.

\section{b. Tierras nacionales}

La mayoría de los ocupantes de tierras nacionales solamente tienen derechos posesorios precarios. No obstante, todavía hay extensiones de terrenos nacionales que no están ocupados y son generalmente de difícil accesibilidad.

Las personas asentadas en tierras nacionales sin conflictividad vecinal solamente tienen derechos posesorios precarios los que, se pueden consolidar por el transcurso del tiempo y bajo el procedimiento para obtener títulos supletorios. Estas personas pueden ser nativas de la región o terceros provenientes de otras regiones del país en busca de tierras para poder fijar su residencia. Categoría aparte son los traficantes de tierras que suelen vender varias veces el mismo lote de terreno incurriendo en delitos penados por la ley. Se identifica un conflicto consistente en una débil presencia del Estado y una riesgosa e insegura adquisición de la propiedad por parte del comprador.

\section{c. Terrenos ejidales}

Existe muy poca documentación sobre los terrenos ejidales. Algunos municipios nacieron en la Costa Caribe Norte asociados a las economías de enclave. Se distinguen dos casos emblemáticos:

1. En el casco urbano del Municipio de Puerto Cabezas no hay tierras ejidales, ya que pertenece en su totalidad al Gobierno Territorial de Karata. Se reconoce al barrio "El Cocal" como una comunidad autónoma y mandata la creación de un mecanismo conjunto para su administración.

2. En Bluefields, los últimos gobiernos municipales se han caracterizado por la donación a particulares de lotes de terrenos localizados en el casco urbano. Tal es el caso de los lotes donados localizados a lo largo de la trocha Nueva Guinea-Bluefields. Se desconoce el asidero legal que sustentan estos actos de enajenación, ya que la ley de municipios y su reglamento sólo contempla donaciones por razones de servicio público.

\section{Propiedad comunal}


Sobre la tenencia de la propiedad, las variantes de propiedad o posesión de tierras en los territorios indígenas se pueden agrupar de la siguiente manera:

- Tierras ocupadas por indígenas en forma ancestral y que, en la actualidad, en su casi totalidad están respaldadas con títulos de territorios indígenas.

- Tierras de terceros privada, adquirida antes de 1987 y que ahora están localizadas dentro de los territorios indígenas.

- Tierras de terceros con respaldo de documentos válidos, que se asentaron en áreas protegidas anteriormente a 1999 y antes de la titulación de territorios indígenas.

- Terceros sin derechos en los territorios indígenas.

La complejidad de la propiedad comunal tiene distintas manifestaciones:

- El número de terceros sobrepasa en mucho el número de comunitarios en varios territorios indígenas: Tasba Pri, Matungbak, Awastigni, Mayagna Sauni Bas y Territorio Rama-Kriol.

- Existe un creciente desfase en la quinta y última etapa del proceso de demarcación y titulación para resolver la situación legal de los terceros que alegan derechos en propiedades comunales. Lo anterior para brindar seguridad jurídica y pleno dominio sobre sus territorios, crucial para la pervivencia y desarrollo de los pueblos y, la atracción de posibles inversionistas.

- Inseguridad jurídica en la contratación ya que usualmente no se respetan los contratos y acuerdos previos, cuando hay cambios de autoridades comunales. Esto suele suceder cuando esas contrataciones se han celebrado violando ciertos mecanismos de legitimación ante la comunidad.

\section{Principales tipos de conflictos}

\section{Conflictos y tensiones en la propiedad privada}

Es muy posible que la mayor conflictividad, en la categoría de propiedad privada, se localice en los municipios donde no hay territorios indígenas. Estos son municipios con un mercado de tierras más dinámico y donde hay menos avance en el ordenamiento de la propiedad rural.

\section{a. Superposición de derechos de propiedad}

Esta situación está mejor documentada en el área rural de Bluefields, donde diversas investigaciones han detectado que una mínima parte de las propiedades están inscritas, según el diagnóstico del Gobierno Territorial Rama y Kriol y que existe una "caprichosa superposición de registros". Los resultados de una de las investigaciones son resumidos de la siguiente manera:

Para la realización de los diagnósticos de Kukra River y Punta Gorda el PROCODEFOR ${ }^{3}$ tuvo que contratar un investigador que buscó en los 272 libros del Registro Público de la Propiedad. Pero las incongruencias entre lo registrado y lo verificado en el campo fueron ostensibles. A partir de los resultados del trabajo de campo realizado en Punta Gorda, PROCODEFOR constató que de las 739 propiedades notificadas que constituyen el 100\% de los datos analizados, solamente 299 estaban legalmente inscritas en el Registro de la Propiedad; y de esas 299, 112 no se pudieron localizar en el terreno, a pesar de aparecer no sólo en el Registro de la Propiedad, sino también en los archivos del Instituto Nicaragüense de Reforma Agraria/Oficina de Titulación Rural (INRA/

\footnotetext{
3 Proyecto de Conservación y Desarrollo Forestal.
} 
OTR), ya sea porque realmente no existían allí donde se decían estar localizados (34 casos) o porque no fue posible ubicarlas en base a la precaria información que en su inscripción presenta el Registro Público (78 casos). (GTR-K, 2007, p. 165).

Un vendedor está obligado a garantizar al comprador la posesión legal de la propiedad; pero, la mayoría de los propietarios no disponen de un título de dominio pleno, de reforma agraria o agrario lo cual se presta a transacciones al margen de la ley. Por tanto, es necesario constatar el estado registral de una propiedad antes de comprar.

\section{b. Litigios por documentos falsos o inapropiados}

Este tipo de conflicto está relacionado con la dramática situación expuesta en los párrafos anteriores. En la medida que un comprador no constate el historial jurídico de una finca en el Registro Público de la Propiedad y que la titularidad de la propiedad esté legalmente definida, habrá más posibilidades de conflictos por documentos falsos o inapropiados.

\section{c. Conflictos por linderos}

La mayoría de las propiedades privadas no están correctamente deslindadas y amojonadas, lo cual a veces es causa de desacuerdos entre vecinos sobre los respectivos linderos. Estos conflictos suelen ser resueltos por medio de una negociación entre las partes o una mediación en el ámbito local. Sin embargo, la capacidad local es limitada, en la mayoría de los municipios, debido a la poca presencia de instituciones que trabajen en la resolución de conflictos en el ámbito local, o el escaso apoyo que reciben las organizaciones locales.

\section{d. Invasión de fincas por personas ajenas}

La mayoría de las veces son sucesos aislados; aunque también hay invasiones que tienen una motivación política partidaria como un procedimiento de presión para obtener dinero y/o bienes o reivindicaciones sociales o políticas. El caso bien conocido es el Territorio de Tasba Pri que colonos han invadido muchos terrenos cuyos dueños están amparados con títulos de reforma agrarias; sin embargo, no lo respetan.

\section{e. Disputas familiares por herencias}

Estas disputas se originan con el fallecimiento de un propietario que no haya testado. Son pocos los casos que llegan a los juzgados para el trámite de declaratoria de herederos, la explicación más plausible es que la mayoría de las disputas son solucionadas en el ámbito local, por medio de acuerdos de buena fe. Esto se presenta con personas no indígenas, ya que como hemos explicado antes, los indígenas tienen mecanismos ancestrales en materia sucesoral.

\section{Conflictos y tensiones en la propiedad pública}

\section{a. Invasión de áreas protegidas por personas no autorizadas}

Las referencias consultadas proveen pistas de que la mayoría de los ocupantes privados de las áreas protegidas son precaristas. Esto incluye numerosas personas que llegaron a las áreas protegidas después de 1999 incluyendo aquellos que tramitaron títulos supletorios o de otro tipo posterior a esa fecha. Muchos terceros en áreas protegidas, han realizado transacciones que, aun cuando medien escrituras públicas, no perfeccionan sus derechos de propiedad.

\section{b. Otorgamiento por autoridades públicas de documentos sin validez legal.}

El artículo 59 del Reglamento de Áreas Prohibidas prohíbe la titulación dentro de estos territorios y establece que son nulos los títulos otorgados o tramitados después de 1999. Un alto porcentaje de los precaristas 
ocuparon tierras con el aval de funcionarios públicos y/o autoridades de los partidos más importantes en el ámbito nacional. El incremento del "caudal electorero" (GTR-K) ha sido la principal motivación detrás de esta desafortunada intervención en las áreas protegidas.

\section{Conflictos y tensiones en la propiedad comunal}

Con la aprobación de la Ley No. 445 se dio una respuesta histórica a las reivindicaciones de los pueblos indígenas para el reconocimiento de sus derechos de propiedad en las tierras que habitaban aún antes de la colonización española e inglesa, y que les fueron usurpadas después de la independencia de Nicaragua con lo que se llamó la "Reincorporación de la Moskitia". Sin embargo, la Ley a falta de regulaciones en materia de competencias y procedimientos para la solución de conflictos ha presentado los siguientes tensionamientos:

En relación a los sujetos beneficiados o perjudicados por esta ley:

a) Conflicto a lo interno de la cada comunidad y entre comunidades por razón de liderazgo y de reparto de las utilidades que podrían generarse en concepto de derechos y aprovechamiento de recursos naturales.

b) Conflicto entre comunidades indígenas de distinto territorios por problemas limítrofes y de extensión de sus territorios.

c) Conflicto entre las comunidades indígenas y los terceros no indígenas que se encuentran ocupando terrenos considerados propios de la comunidad indígena. Los terceros no indígenas, sobre todo los nacidos en la región se sienten con derechos posesorios en terrenos indígenas.

d) Conflictos entre terceros no indígenas por reclamaciones de derechos sobre tierras consideradas propiedad de los indígenas. Los terceros no indígenas, no sólo tienen conflictos con la comunidad indígena, si no entre ellos mismos por el control precario de la tierra.

En relación con los diferentes entes del Estado:

a. Conflictos de las comunidades indígenas con los diferentes órganos y poderes del Estado directamente involucrados en el cumplimiento de la ley $\otimes_{445}$ en relación a:

i. Certificación de las elecciones de sus líderes y registro de las mismas. Desde los Consejos Regionales, los grupos partidarios imponen las autoridades territoriales desnaturalizando el sentido de la ley; ya que ellos sólo deben emitir las certificaciones correspondientes.

ii. Otorgamiento de concesiones o contratos de aprovechamiento de recursos naturales en territorios indígenas. Se presentan conflictos cuando se ha manipulado la voluntad de la comunidad sustituyéndola por avales que no cuenta con la aprobación de la Asamblea Comunal.

iii. La distribución de los tributos recaudado por el fisco en concepto de derechos de aprovechamiento de recursos naturales en territorios indígenas lo cual genera conflicto entre los líderes.

iv. La declaración de áreas protegidas en propiedades comunales y administración de las mismas ya que no existen mecanismos claros de la participación de las autoridades comunitarias en el manejo responsable de estas áreas.

\section{En relación con la gestión de las distintas comisiones creadas por ley:}

Las comunidades muchas veces se sienten desprotegidas frente a las instituciones estatales que tienen formas administrativas de operar que los líderes no entienden, y en muchas ocasiones no comparten por 
ser ajenos a sus prácticas ancestrales. Se les obliga a pasar por procedimientos administrativos engorrosos que vuelve interminable el proceso.

\section{a. Autoridades Municipales, Regionales, Gobierno Central, Ministerio de Recursos Naturales, Agropecuario y Oficina de Titulación Rural}

Se carece de mecanismos para la coordinación, colaboración y cooperación entre los diferentes órganos de Gobierno Regionales y Municipales; de estos con el Gobierno Central e instituciones estatales nacionales $\mathrm{y}$ todos ellos con las autoridades comunales y territoriales.

En relación al proceso de Demarcación y Titulación el conflicto se ha presentado entre las comunidades y de estas con los terceros y demás órganos del Estado en las diferentes etapas previstas en el artículo 45 de la ley 445 .

Conflictos que no han concluido y se seguirá manifestando en la etapa de saneamiento; principalmente por falta de consenso con las autoridades comunales sobre cómo debe abordarse el conflicto para solucionarlo; por la prevalencia de intereses partidarios ajenos a los intereses de la comunidad; por la falta de disponibilidad de recursos destinados a dar terminado con el proceso de demarcación territorial.

En este contexto, no todo ha sido negativo. Los líderes de los territorios indígenas han elaborado políticas de convivencia que orientan los procedimientos para la estadía de terceros en sus tierras. Estas políticas han sido socializadas con líderes y comunitarios que han venido ingresando a sus territorios en los últimos años. En general, podemos indicar que el régimen jurídico de la tenencia de la tierra es bastante claro, no así la competencia y procedimientos para la solución de conflictos. Esto ocasiona que los procesos sean más complejos y persistan atrasos para dar solución a los mismos.

\section{b. Conflictos con ocupantes precaristas}

Constituyen la mayoría de los terceros en los territorios indígenas. Esto incluye poseedores de escrituras públicas de compraventa de propiedad comunal que son nulos. Existe una diversidad de razones por las cuales hay un avance lento en este aspecto fundamental de la etapa de saneamiento, incluyendo la ausencia de un consenso político. Es previsible que haya un gran descontento social de los afectados e inclusive violencia, al momento del desalojo masivo, dado el mayor o menor grado de inversiones realizados por los afectados.

\section{c. Conflictos con terceros que tienen documentos emitidos por síndicos y comunitarios de forma irregular.}

Este estudio ha revelado que un significativo porcentaje de los precaristas en territorios indígenas argumentan que tienen "papelitos" en su poder, pues hicieron transacciones de compraventa o arrendamientos ilegales con síndicos y comunitarios. Tema que los líderes indígenas entrevistados prefieren no discutir.

\section{Incertidumbre sobre la indemnización a poseedores de títulos agrarios con algún vicio de forma o de fondo}

Es definida en el artículo 38 de la ley 445. No se conoce de un diagnóstico de esta situación en la Costa Caribe. No está claro cuál será la fuente de financiamiento de la indemnización para que estos titulares de reforma agraria abandonen el territorio indígena.

Una síntesis de los niveles de complejidad y tipos de conflictividad que se presentan en los diferentes tipos de tenencia de tierra es la que se refleja en el siguiente mapa donde se nota la sobreposición geográfica de los diferentes tipos de tenencia. 


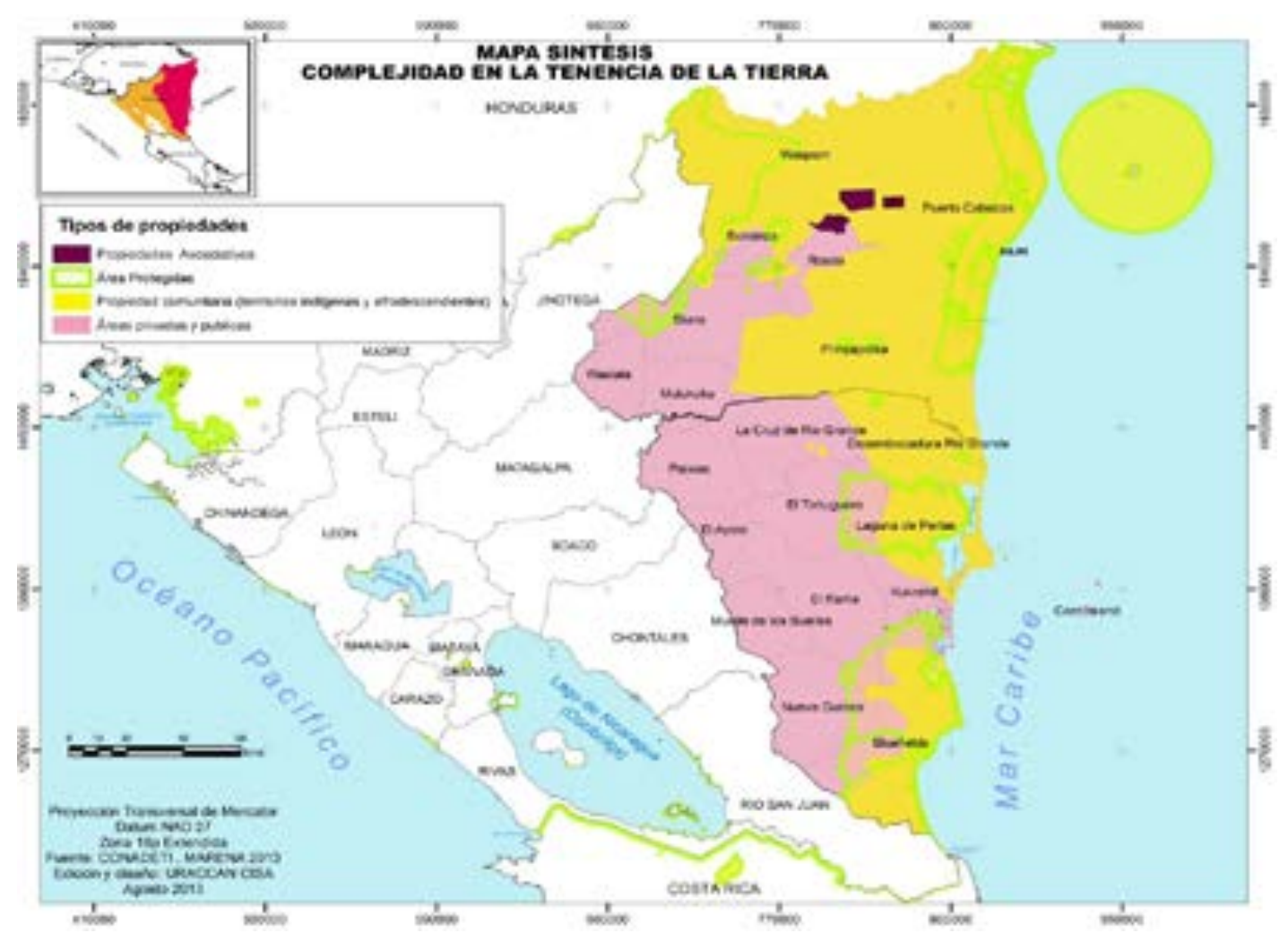

Mapa. Niveles de complejidad y tipos de conflictividad en los diferentes tipos de propiedad.

Fuente: Elaboración propia con base a información de la consultoría.

\section{Conclusiones}

Si bien, la Constitución Política de Nicaragua reconoce, protege y promueve las distintas formas de propiedad, en la práctica se hace necesario un uso eficiente de los recursos asignados para el proceso de legalización de la tierra por parte de las instancias gubernamentales con presencia en la Costa Caribe de Nicaragua.

Al no existir un inventario y registro actualizado de la propiedad en la Costa Caribe de Nicaragua dificulta los negocios entre particulares, y aumenta los riesgos en la inversión puesto que no se puede tener certeza de la legítima titularidad del que afirma tenerla. Esta situación trae como consecuencia, litigios por documentos falsos o ineficaces; emisión de títulos nulos por autoridades públicas no legitimadas para hacerlo; superposición (traslape) de supuestos derechos sobre un mismo fundo; conflictos por linderos; invasión de colonos y precaristas en propiedades privadas; disputas familiares por razones de herencia, entre otros.

La más conocida en las Regiones Autónomas es la propiedad comunal que goza de una protección especial estatal. No obstante, las principales complejidades son las siguientes:

1. La creciente población no indígena en territorios indígenas, tensiona en muchas ocasiones la relación entre ellos o con otros que llegan a querer ocupar los terrenos que ellos tienen de forma ilegal y todos ellos con los indígenas. El punto más alto en este tensionamiento gira alrededor de la tenencia de la tierra y sus recursos y en muchas ocasiones ha desembocado en quemas de viviendas, destrucción de siembras, lesiones y muertes. 
2. La politización y partidización en la elección de las autoridades comunitarias potencia la inseguridad jurídica en la contratación, porque las nuevas autoridades electas muchas veces no reconocen ni respetan los acuerdo o convenios realizados con las anteriores autoridades. También son frecuentes las divergencias en el reparto de las utilidades.

3. El desfase y la falta de conclusión de la etapa de saneamiento impide la emisión de los títulos de propiedad a favor de las comunidades indígenas y consecuentemente dificulta las negociaciones para la inversión.

De manera general, todas las formas de tenencia de la tierra en la Costa Caribe nicaragüense están limitadas en su ejercicio ya sea por utilidad pública e interés social o por leyes especiales que regulan las áreas protegidas, terrenos fronterizos y costeros. También están reguladas según el giro del negocio o uso que se le pretenda dar; para el caso de la Costa Caribe nicaragüense solamente de observar y atender la cultura del lugar y las disposiciones legales vigentes.

\section{Agradecimiento}

Esta publicación obtuvo el financiamiento de: El Fondo de Asistencia Internacional de los Estudiantes y Académicos Noruegos (SAIH).

\section{Lista de referencias}

Cabanellas de Torres, Guillermo (1992). Diccionario de Ciencias Jurídicas, Políticas y Sociales. 20ma. Edición, Editorial Heliasta. Buenos Aires, Argentina.

Declaración Universal de los Derechos Humanos, Naciones Unidas, 1948.

\section{GACETA, DIARIO OFICIAL}

Código Civil de la República de Nicaragua, Managua, Nicaragua, 1904.

Constitución Política de Nicaragua, Managua, Nicaragua, 2014.

Estatuto de Autonomía de las Regiones Autónomas de la Costa Atlántica de Nicaragua, Ley No. 28, Managua, Nicaragua, 1987.

Ley de Régimen Propiedad Comunal de los Pueblos Indígenas y Comunidades Étnicas de las Regiones Autónomas de la Costa Atlántica de Nicaragua y los ríos Bocay, Coco e Indio Maíz, Ley No. 445, Managua, Nicaragua, 2003. 